\title{
Cardiac Function and Microsphere Blood Flow Distribution in the Brain-Dead Dog
}

\author{
Thomas S. Huber, Bradley J. Martin, Susan M. Stante, and Louis G. D'Alecy
}

\begin{abstract}
The mechanisms for the deterioration of the braindead organ donor are not clearly defined. We measured myocardial blood flow and function during the 4 hours after the induction of brain death in dogs. Brain death was induced by elevating and maintaining intracranial pressure above systolic arterial pressure and effectively stopping central nervous system blood flow. Multiorgan blood flow and systemic arteriovenous shunting were measured using radioactive microspheres. The mean arterial pressure was found to decrease markedly with the induction of brain death. The initial changes in mean arterial pressure were attributed to a decrease of systemic vascular resistance, with the more terminal changes due to a decrease in cardiac index. There was a marked decrease
\end{abstract}

T

HE ADVANCES in the ficld of solid organ transplantation over the past decade have emphasized the need for a better understanding of the physiology and pathophysiology of the clinical brain-dead state, one lacking all cortical, brain stem, and hypothalamic function. Clearly, improved donor management could potentially expand the organ pool and favorably impact immediate organ function.

The mechanisms for the progression from clinical brain death to somatic death are unknown. It is known that this progression occurs within in a finite period of time which is usually measured within hours and that this progression occurs despite maximal clinical therapy, including mechanical ventilation, inotropes, and pressor agents. The majority of our understanding has been provided by retrospective or uncontrolled clinical studies; however, several recent laboratory studies have been reported and have focused attention on the myocardium as the

From the Departments of Physiology and Surgen, The University of Michigan Medical School, Ann Arbor, MI.

Received June 3, 1991; accepted December 1, 1991.

Supported in part by the American Heart Association of Michigan. Dr Huber was a recipient of a National Research Service Award Fellowship from National Heart, Lung, and Blond Institute, sponsor no. 5-F32-HI.07920-n2.

Address reprint requests to Louis G. D'Alecy, DMD, PhD, Department of Physiology, The University of Michigan Medical School, M7799 Medical Science II, 1301 Catherine St, Ann Arbor, MI 48109-0622.

Copyright $\odot 1992$ by W.B. Saunders Company

0883-9441/92/0702-0003\$05.00/0 of left ventricular $\mathrm{dP} / \mathrm{dt}$ with the induction of brain death and a gradual decrease of stroke volume despite no change in pulmonary capillary wedge pressure. The microsphere calculated blood flows to the left ventricle and septum of the myocardium were significantly lower at the 1- and 4-hour time points relative to control. However, the coronary sinus oxygen extraction ratio was not statistically different from control at 4 hours. Systemic arteriovenous shunts increased after the induction of brain death but remained below $10 \%$. We conclude that despite brain death-induced hypotension, there is little evidence to suggest that marked myocardial hypoxic ischemic changes initiated the deterioration in this model.

Copyright $\odot 1992$ by W.B. Saunders Company

primary factor. Novitzky et al $^{1}$ have reported the electrocardiographic changes that occur after the induction of brain death in a primate model and have characterized several specific stages of deterioration. Furthermore, Novitzky et $\mathrm{al}^{2,3}$ documented the histologic changes occurring in the myocardial fibers after brain death and were able to prevent these changes with sympathectomy and pharmacologic agents. Wicomb et $\mathrm{al},{ }^{4}$ using hearts excised from a brain-dead pig model on a working heart preparation, reported a decrement of myocardial performance. Additionally, Sasaki et al ${ }^{5}$ reported a decrement of myocardial contractility after the induction of brain death in a canine model using the left ventricular pressurevolume loop relationship.

Although the available experimental data suggest that there is a decrement of myocardial performance after the induction of brain death, the literature is difficult to integrate on several accounts. There has been variability in both the species and the techniques for producing brain death. The animal models have varied in both the magnitude and duration of the Cushing reflex intrinsic to the induction of brain death and in the assessment of the completeness of brain death. Additionally, the traditional therapies of fluid, pressors, and inotropes have often been used to maintain presumed normal arterial pressures and organ perfusion. However, it has not been proven that these strategies and the principles of normal regulatory physiology 
are appropriate in this setting; it is conceivable that the current strategies are detrimental.

The purpose of this study was threefold: to determine the hemodynamic changes in a severe, well-controlled model of the potential organ donor in the presence of only fluid support, to determine the changes in regional blood flow distribution after the induction of brain death, and to determine whether myocardial hypoxic ischemia contributed to the observed decrement of myocardial performance.

\section{MATERIALS AND METHODS}

\section{General}

The standard preparation has been previously reported ${ }^{6}$ and will be only briefly summarized. Seventeen fasted adult male mongrel dogs weighing from 15 to $25 \mathrm{~kg}$ were anesthetized with $17.5 \mathrm{mg} / \mathrm{kg}$ of intravenous thiamylal sodium (Surital; Parke-Davis, Morris Plains, NJ) and supplemented as needed until the induction of brain death. The animals were intubated and ventilated (Harvard 607, South Natick, $\mathrm{MA}$ ) with room air. Plasma $\mathrm{pH}$ was determined throughout the experiment (IL $113 \mathrm{pH} /$ Blood Gas Analyzer, Lexington, MA) and normalized with supplemental intravenous sodium bicarbonate $(1.5 \%)$ and ventilator changes. A heating pad and lamps were used to minimize cooling. Two 18gauge needles were placed through the atlanto-occipital membrane into the cisterna magna for pressure monitoring and saline infusion. The femoral artery was cannulated for continuous measurement of pulsatile and mean arterial pressure (MAP) and as a reference site for the microsphere withdrawal. A flow-directed, balloon-tipped catheter (SwanGanz; Oximetrix, Mountain View, CA) was advanced from the femoral vein into the pulmonary artery for measurement of pulmonary capillary wedge pressure (PCWP), central venous pressure, and cardiac output. Normal saline $(1.5 \mathrm{~L})$ was administered through the Swan-Ganz catheter during instrumentation. A nylon suture snare was placed around the inferior vena cava (IVC) just caudal to the pericardium. A high-fidelity pressure transducer (Koningsberg, Pasadena, CA) was placed into the left ventricle through the apex for continuous measurement of left ventricular pressure and its first derivative $\mathrm{dP} / \mathrm{dt}(\mathrm{N}=7)$. Catheters were placed into the ascending aorta for the microsphere reference withdrawal and into the left atrial appendage for the microsphere infusion. After the appropriate hemodynamic control values were recorded and the control microsphere infusion performed, brain death was induced as described below. Microsphere infusions were repeated at 1 and 4 hours post-brain death. Cardiac index (CI) was determined by dividing the cardiac output by the body surface area. Systemic vascular resistance (SVR; dynes $\cdot \mathrm{sec} / \mathrm{cm}^{5} / \mathrm{m}^{2}$ ) was determined by dividing the difference of the MAP and the central venous pressure by the $\mathrm{CI}$ and multiplying by a factor of 80 . Tissue blood flow $(\mathrm{N}=10)$ was calculated using the radioactive microsphere technique previously described ${ }^{7.8}$ and briefly outlined here. Approximately $2 \times$ $10^{6} \quad 15-\mu \mathrm{m}$ radiolabeled microspheres (randomly chosen tin-113, cerium-141, or scandium-46) (New England $\mathrm{Nu}-$ clear, Boston, MA) were infused into the left atrial appendage. At autopsy, 33 systemic tissues were harvested from consistent anatomic locations. These tissues were sectioned, weighed, desiccated, and counted in the gamma counter (Packard 5330 Gamma Scintillation Spectrometer, Downers Grove, IL). The experiment was terminated after the final microsphere infusion, and the animals were given 120 $\mathrm{mg} / \mathrm{kg}$ of intravenous pentobarbital (Uthol; Butler $\mathrm{Co}$. Columbus, $\mathrm{OH}$ ) to ensure adequate euthanasia.

\section{Brain Death}

Approximately $7 \mathrm{~mL}$ of freshly drawn, nonheparinized blood was slowly infused into the cisterna magna and allowed to clot for several minutes. The snare on the IVC was then tightened to reduce venous return and decrease arterial pressure. When the systolic arterial pressure decreased to approximately $50 \mathrm{~mm} \mathrm{Hg}$, the intracranial pressure was elevated above it and maintained at least 20 $\mathrm{mm} \mathrm{Hg}$ above it throughout the duration of the experiment. Intracranial pressure was elevated above the systolic arterial pressure within 5 seconds. An isoelectric electroencephalogram resulted at this point and brain death was declared. The initial rise in intracranial pressure precipitated a marked, but transient, elevation of the arterial pressure from the Cushing reflex. The reflex increase in arterial pressure was limited by the reduced venous return from the snare on the IVC, and a second fall in the arterial pressure to $50 \mathrm{~mm} \mathrm{Hg}$ resulted within 1 minute. The snare on the IVC was removed at this point, thus augmenting venous return and producing a rise in arterial pressure. An initial steady-state condition resulted approximately 5 minutes after the declaration of brain death. The absence of the central nervous system (CNS) function and blood flow have been reported. ${ }^{\circ}$

\section{Systemic Arteriovenous Shunts}

The percentage of the cardiac output passing through systemic arteriovenous shunts was determined in a subset of the animals in which the microsphere analysis was performed $(\mathrm{N}=5)$. At the time of autopsy both lungs were harvested. They were carbonized by baking at $325^{\circ} \mathrm{C}$ for 24 hours and were then counted in the gamma counter." The calculated blood flow to the lung was expressed as a percentage of cardiac output. All microspheres reaching the pulmonary circulation were presumed to pass through systemic arteriovenous shunts. A small error from bronchial artery blood flow was unavoidable with this technique and would tend to give falsely high calculated shunt values.

\section{Coronary Sinus Oxygen Extraction}

In a subset of seven dogs, a catheter was placed into the coronary sinus through the right atrial appendage and advanced circumferentially around the heart to the juxtaposition of the left atria and the coronary sinus. Blood was sampled simultaneously from the aorta and the coronary sinus at selected time points. The oxygen content in the samples was determined with a fuel cell oxygen analyzer (LEXO ${ }_{2} \mathrm{CON}$; Lexington Instruments, Hospex, Chestnut Hill, MA). The percent coronary sinus oxygen extraction 
was determined by dividing the difference between the coronary sinus oxygen content and the aortic oxygen content by the aortic oxygen content.

\section{Statistical Analyses}

The hemodynamic and physiologic variables, the percentage of arteriovenous shunts, the calculated microsphere flow values, and the coronary sinus oxygen extraction values were analyzed with an ANOVA test. Scheffe's confidence intervals were used to determine the statistically significance differences; a level of $P<.1$ was accepted as statistically significant. The microsphere data analysis was facilitated using the Michigan Interactive Data Analysis System software of The University of Michigan Statistical Research Laboratory on an IBM 3090 (New York, NY) computer. All other statistical analyses were performed on a Macintosh 11 (Apple Computer Inc, Cupertino, CA) using the Statview 512 (Brain Power Inc, Calabasas, CA) software.

\section{Experimental Approval}

The experimental procedure conformed to the guidelines established by the American Physiological Society and the National Institutes of Health (Guide for the Care and Use of Laboratory Animals, NIH Publication no. 85-23,1985), and was approved by The University of Michigan Unit for Laboratory Animal Medicine's Vertebrate Animal Use Committee (approval no. 1677A).

\section{RESULTS}

The baseline hemodynamics and patterns of change were similar in all 17 dogs. These data are presented as a single group with subgroups for microsphere flow $(\mathrm{N}=10)$, coronary sinus oxygen extraction $(\mathrm{N}=7), \mathrm{dP} / \mathrm{dt}(\mathrm{N}=7)$, and arteriovenous shunt measurements $(\mathrm{N}=5)$ identified as appropriate for the reduced sample sizes. The major time points analyzed corre- sponded to the three measurements of microsphere blood flow: the control prior to brain death, 1 hour post-brain death, and 4 hours post-brain death.

The mean operative duration measured from the induction of anesthesia to the induction of brain death was $135 \pm 10$ minutes (mean \pm 1 SEM) and the mean anesthetic dose was $32 \pm 2$ $\mathrm{mg} / \mathrm{kg}$. The mean volume of normal saline administered was $80 \pm 2 \mathrm{~mL} / \mathrm{kg}$. The hemodynamic and physiologic variables at the time of microsphere infusion are presented in Table 1. The small SEM for control MAP, CI, heart rate (HR), and PCWP suggest that the fluid balance on completion of the instrumentation was similar across all animals. Despite the use of heat pad and lamps, there was a slight decrease of temperature after the induction of brain death. Plasma glucose fell after brain death and continued to decrease throughout the duration of the experiment; these changes reached statistical significance with a greater than $50 \%$ reduction in blood glucose at 4 hours relative to control.

The initial elevation of intracranial pressure resulted in a variety of transient arrythmias that resolved within 5 minutes after the induction of brain death. These included sinus tachycardia, supraventricular tachycardias, sinus bradycardia, and nodal arrythmias. No specific pattern was detected. After this brief period, a normal sinus rhythm was detected throughout the remaining duration of the experiment without

Table 1. Hemodynamic and Physiologic Parameters

\begin{tabular}{|c|c|c|c|}
\hline Parameter & Control & 1 Hour Post-BD & 4 Hours Post-BD \\
\hline MAP $(\mathrm{mm} \mathrm{Hg})$ & $117 \pm 4$ & $67 \pm 4^{*}$ & $59 \pm 4^{*}$ \\
\hline $\mathrm{Cl}\left(\mathrm{I} / \mathrm{min} / \mathrm{m}^{2}\right)$ & $4.67 \pm 0.32$ & $4.87 \pm 0.38$ & $2.70 \pm 0.20^{*}, \dagger$ \\
\hline SVR (dynes $/ \mathrm{s} / \mathrm{cm}^{5} / \mathrm{m}^{2}$ ) & $2,055 \pm 131$ & $1,088 \pm 79^{*}$ & $1,698 \pm 81^{*}, \dagger$ \\
\hline HR (beats/min) & $150 \pm 8$ & $131 \pm 2^{*}$ & $126 \pm 3^{*}$ \\
\hline SV (I) & $0.034 \pm 0.003$ & $0.037 \pm 0.003$ & $0.021 \pm 0.002^{*}, \dagger$ \\
\hline $\mathrm{PCWP}(\mathrm{mm} \mathrm{Hg})$ & $8.4 \pm 0.6$ & $9.0 \pm 0.7$ & $7.9 \pm 0.6$ \\
\hline $\mathrm{Dp} / \mathrm{dt}(\mathrm{mm} \mathrm{Hg} / \mathrm{s})$ & $2,161 \pm 136$ & $1,457 \pm 115^{*}$ & $992+88^{*}, t$ \\
\hline Temperature $\left({ }^{\circ} \mathrm{C}\right)$ & $38.2 \pm 0.2$ & $37.2 \pm 0.2^{*}$ & $37.5 \pm 0.4^{*}, \dagger$ \\
\hline $\mathrm{pH}(\mathrm{U})$ & $7.36 \pm 0.02$ & $7.34 \pm 0.02$ & $7.37 \pm 0.01 \dagger$ \\
\hline Glucose (mg/dL) & $125 \pm 6$ & $98 \pm 6^{*}$ & $60 \pm 5^{*}, T$ \\
\hline
\end{tabular}

Note. The hemodynamic and physiologic parameters are reported as the mean values \pm 1 SEM for the control, 1- and 4-hour time points. The values were analyzed with an ANOVA test and Scheffe's confidence intervals were used to determine statistical significance of the differences. A level of $P \leq .1$ was accepted as statistically significant.

Abbreviations: BD, brain death; MAP, mean arterial pressure; $\mathrm{Cl}$, cardiac index; SVR, systemic vascular resistance; $\mathrm{HR}$, heart rate; $\mathrm{SV}$, stroke volume; PCWP, pulmonary capillary wedge pressure; Dp/dt, first derivative of left ventricular pressure.

* Statistical difference between the control value.

tStatistical difference between the 1-hour value. 


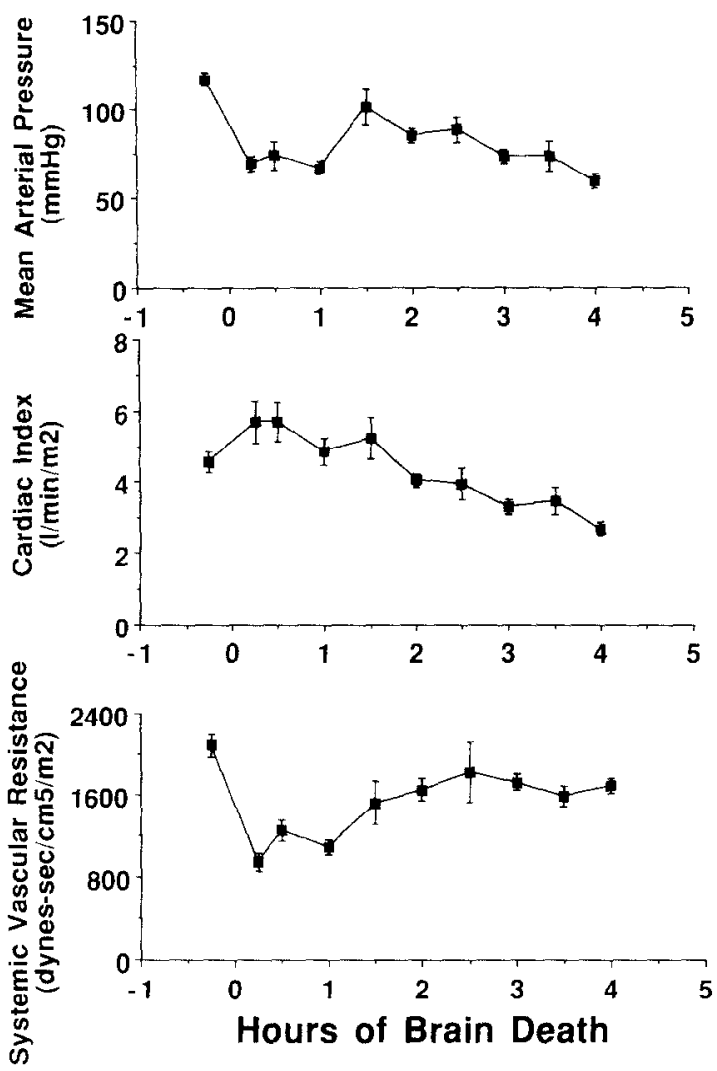

Fig 1. The changes in MAP (mm $\mathrm{Hg}), \mathrm{Cl}\left(\mathrm{L} / \mathrm{min} / \mathrm{m}^{2}\right)$, and SVR (dynes $\cdot \mathrm{sec} / \mathrm{cm}^{5} / \mathrm{m}^{2}$ ) are graphed against the hours of brain death. The induction of brain death corresponds to the zero time point. The error bars correspond to \pm 1 SEM

further evidence of ectopy or electrocardiographic ischemic changes.

The pattern of changes in MAP, CI, and SVR are presented in Fig 1. After the induction of brain death, there was a marked decrease in MAP. This decrease was predominantly due to a decrease of the SVR at the 1-hour point and to a combined decrease of the CI and SVR at the 4-hour point. The $\mathrm{CI}$ was not different from the control value at 1 hour, but it continued to fall, reaching significance at 4 hours. This decrease in $\mathrm{CI}$ was seen despite no change in the PCWP and a decreasing MAP. The SVR was markedly decreased during the first hour, but gradually increased thereafter; it never fully returned to the control value.

The components of the $\mathrm{CI}$ can be further examined. The HR and stroke volume (SV) versus time are presented in Fig 2. On the induction of brain death, there was a marked decrease of HR with little subsequent change. The calculated SV actually increased immedi-

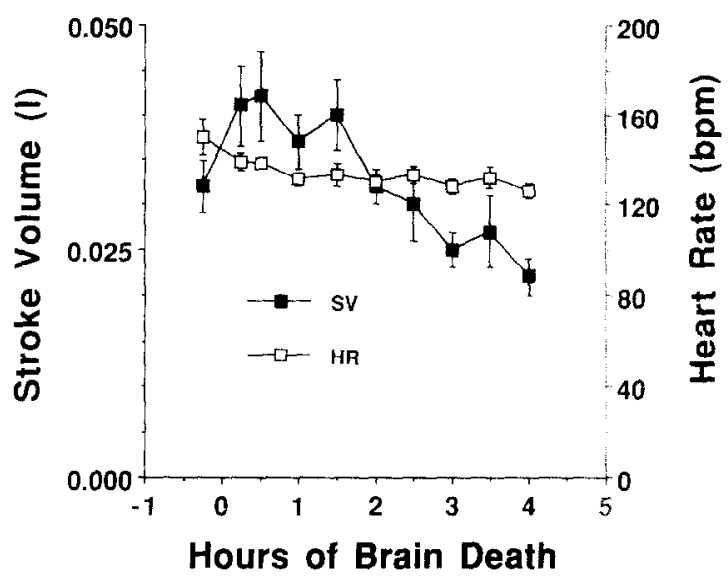

Fig 2. The changes in HR (beats/min) and SV (L) are graphed against the hours of brain death. The induction of brain death corresponds to the zero time point. The error bars correspond to \pm 1 SEM.

ately after brain death, but fell off after the 1-hour point as would be predicted from the CI. The $\mathrm{dP} / \mathrm{dt}$ is plotted against time in Fig 3. There was a marked decrease of $\mathrm{dP} / \mathrm{dt}$ after the induction of brain death, with progressive deterioration thereafter. The progressive decrease in $\mathrm{SV}$ after 1.5 hours parallels $\mathrm{dP} / \mathrm{dt}$.

The microsphere calculated blood flow values for the three time points are contained in Table 2 . All the left ventricular and septal flows were found to be statistically lower at the 1 - and 4-hour points relative to control. No difference in flow was found between the 1-and 4-four hour points, however. A similar pattern of flow changes was seen for the thyroid and adrenal glands. No statistical changes in flow were noted

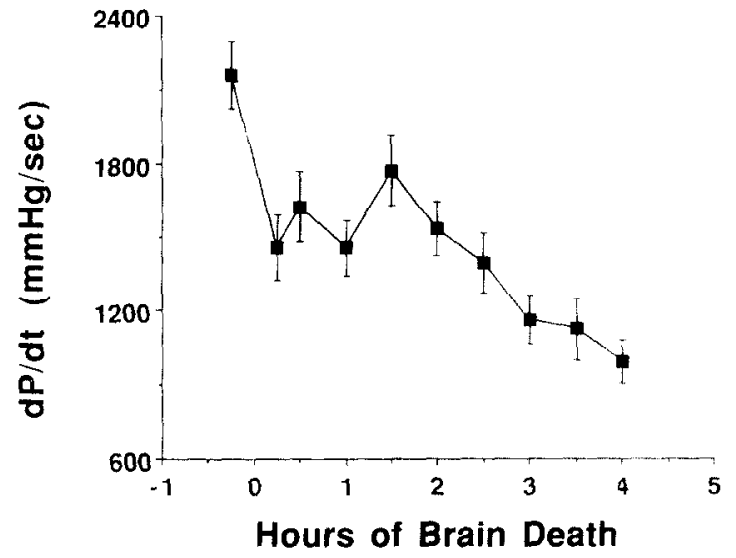

Fig 3. $\mathrm{DP} / \mathrm{dt}$ (mm $\mathrm{Hg} / \mathrm{s}$ ) is graphed against the hours of brain death. The induction of brain death corresponds to the zero time point. The error bars correspond to \pm 1 SEM. 
Table 2. Calculated Microsphere Flows $(\mathrm{mL} / \mathrm{min} / 100 \mathrm{~g})$

\begin{tabular}{|c|c|c|c|}
\hline Tissue & Contral & 1 Hour Post-BD & 4 Hours Post-BD \\
\hline \multicolumn{4}{|l|}{ Heart } \\
\hline LV epicardium & $124.5 \pm 20.6$ & $77.0 \pm 5.2^{*}$ & $43.3 \pm 4.3^{*}$ \\
\hline LV mid wall & $140.6 \pm 19.0$ & $83.2 \pm 7.7^{*}$ & $47.9 \pm 5.1^{*}$ \\
\hline LV endocardium & $158.8 \pm 21.5$ & $91.2 \pm 6.7^{*}$ & $51.7 \pm 6.3^{*}$ \\
\hline Septum & $131.9 \pm 16.0$ & $76.4 \pm 6.7^{*}$ & $46.8 \pm 4.5^{*}$ \\
\hline RV & $83.9 \pm 9.3$ & $62.7 \pm 6.5$ & $40.6 \pm 5.2^{*}$ \\
\hline $\mathrm{R}$ atrium & $36.0 \pm 6.2$ & $44.1 \pm 9.3$ & $15.6 \pm 2.1 \dagger$ \\
\hline \multicolumn{4}{|l|}{ Lung } \\
\hline$R$ upper lobe & $2.6 \pm 1.6$ & $28.3 \pm 10.1$ & $20.9 \pm 11.4$ \\
\hline A lower lobe & $8.0 \pm 3.2$ & $50.9 \pm 21.5$ & $38.3 \pm 14.5$ \\
\hline \multicolumn{4}{|l|}{ Liver } \\
\hline R lobe distal & $25.9 \pm 6.4$ & $15.4 \pm 3.2$ & $6.7 \pm 2.3^{*}$ \\
\hline$R$ lobe parenchymal & $35.0 \pm 7.3$ & $26.4 \pm 5.9$ & $9.6 \pm 2.5^{*}$ \\
\hline L lobe distal & $29.2 \pm 7.2$ & $20.3 \pm 3.9$ & $6.1 \pm 1.4^{*}$ \\
\hline L lobe parenchymal & $38.3 \pm 10.6$ & $29.3+7.7$ & $9.6 \pm 2.9^{*}$ \\
\hline \multicolumn{4}{|l|}{ Kidney } \\
\hline R outer cortex & $602.3 \pm 43.8$ & $422.6 \pm 19.0^{*}$ & $308.1 \pm 28.1^{*}, \dagger$ \\
\hline R inner cortex & $249.3 \pm 59.2$ & $210.5 \pm 42.6$ & $114.9 \pm 24.4$ \\
\hline R medulla & $50.3 \pm 27.9$ & $35.6 \pm 15.2$ & $26.8 \pm 14.9$ \\
\hline L outer cortex & $583.6 \pm 46.7$ & $389.8 \pm 19.6^{*}$ & $289.6 \pm 20.9^{*}$ \\
\hline$L$ inner cortex & $240.1 \pm 62.0$ & $206.7 \pm 46.3$ & $103.4 \pm 21.9$ \\
\hline L medulla & $34.6 \pm 11.8$ & $23.9 \pm 6.8$ & $17.8 \pm 7.9$ \\
\hline Pancreas & $40.6 \pm 7.6$ & $61.1 \pm 11.9$ & $25.9 \pm 4.4 \dagger$ \\
\hline Spleen & $102.5 \pm 20.1$ & $61.4 \pm 10.0$ & $25.5 \pm 6.8^{*}$ \\
\hline \multicolumn{4}{|l|}{ Stomach } \\
\hline Serosa & $30.4 \pm 24.6$ & $5.4 \pm 1.5$ & $5.3 \pm 2.4$ \\
\hline Mucosa & $92.5 \pm 25.0$ & $34.9 \pm 6.5^{*}$ & $28.4 \pm 3.3^{*}$ \\
\hline \multicolumn{4}{|l|}{ Small intestine } \\
\hline Serosa & $22.9 \pm 4.0$ & $16.8 \pm 2.8$ & $10.5 \pm 3.3^{*}$ \\
\hline Mucosa & $184.1 \pm 25.4$ & $82.4 \pm 7.6^{*}$ & $73.4 \pm 5.0^{*}$ \\
\hline \multicolumn{4}{|l|}{ Colon } \\
\hline Serosa & $61.5 \pm 26.8$ & $48.3 \pm 19.7$ & $18.6 \pm 4.5$ \\
\hline Mucosa & $133.7+32.4$ & $164.5 \pm 41.0$ & $98.3 \pm 18.3$ \\
\hline R adrenal & $326.6 \pm 45.5$ & $126.2 \pm 13.8^{*}$ & $96.9 \pm 9.3^{*}$ \\
\hline L adrenal & $348.5 \pm 42.0$ & $129.0 \pm 13.6^{*}$ & $105.8 \pm 11.6^{*}$ \\
\hline $\mathrm{R}$ thyroid & $159.2 \pm 30.1$ & $29.3 \pm 3.9^{*}$ & $15.6 \pm 2.2^{*}$ \\
\hline$L$ thyroid & $229.7 \pm 23.6$ & $43.5 \pm 5.61^{*}$ & $23.2 \pm 3.4^{*}$ \\
\hline Skeletal muscle & $3.1 \pm 0.3$ & $5.2 \pm 0.8^{*}$ & $3.0 \pm 0.4 \dagger$ \\
\hline Skin & $4.7 \pm 0.9$ & $4.9 \pm 0.9$ & $3.0 \pm 0.6$ \\
\hline Tongue & $2.1 \pm 0.4$ & $14.9 \pm 1.7^{*}$ & $7.2 \pm 1.5^{*}, \dagger$ \\
\hline
\end{tabular}

Note. The calculated microsphere flow values are reported in $\mathrm{mL} / \mathrm{min} / 100 \mathrm{~g}$ tissue for the control, 1- and 4-hour time points. The values are reported as the mean $\pm 1 \mathrm{SEM}$. The tissues are reported by their anatomic location. The values were analyzed with an ANOVA test and Scheffe's confidence intervals were used to determine statistical significance of the differences. $P \leq .1$ was accepted as statistically significant.

Abbreviations: LV, left ventricular; RV, right ventricle; $R$, right; $L$, left.

*Statistical difference between the control value.

tStatistical difference between the 1-hour value.

for the pulmonary tissues despite a tendency to increase in the calculated mean values. The liver tissues harvested all showed the same pattern of flow changes; the mean flow values decreased after brain death, but the only statistical decrease was found between the control and 4-hour time point. The calculated flows for the right and left kidney outer cortices were found to decrease after brain death, reaching statistical significance for the 1- and 4-hour time points relative to control. No statistical difference in the flow was found for the other harvested, anatomic sections of the kidney despite a decreasing trend. Skeletal muscle and the tongue were the only tissues to show a statistical rise in the calculated flow at the 
1-hour time point relative to control; both tissues had a decrease in flow between the 1and 4-hour points, which reached statistical significance. A graphic representation of the changes in flow expressed as a percentage of the control value is contained in Fig 4 for selected heart, liver, kidney, tongue, and skeletal muscle sections.

The coronary sinus oxygen extraction shown in Fig 5 was $71.5 \% \pm 4.5 \%$ at the control measurement and increased slightly to $76.9 \% \pm$ $2.6 \%$ at the 4-hour time point. The control value was not found to be statistically different from any of the values after brain death.

The calculated arteriovenous shunts based on the whole lung counts are expressed as a percentage of the cardiac output that passed through channels larger than $15 \mu \mathrm{m}$. The shunts increased markedly from $1.2 \% \pm 0.2 \%$ at control to $8.0 \% \pm 1.2 \%$ at 1 hour and $9.3 \% \pm 2.1 \%$ at 4 hours. A statistically significant increase was found between the control value and the 1- and 4-hour values.

\section{DISCUSSION}

The present study indicates that the induction of brain death in this severe model of the potential organ donor leads to a decrement of myocardial performance in the absence of a

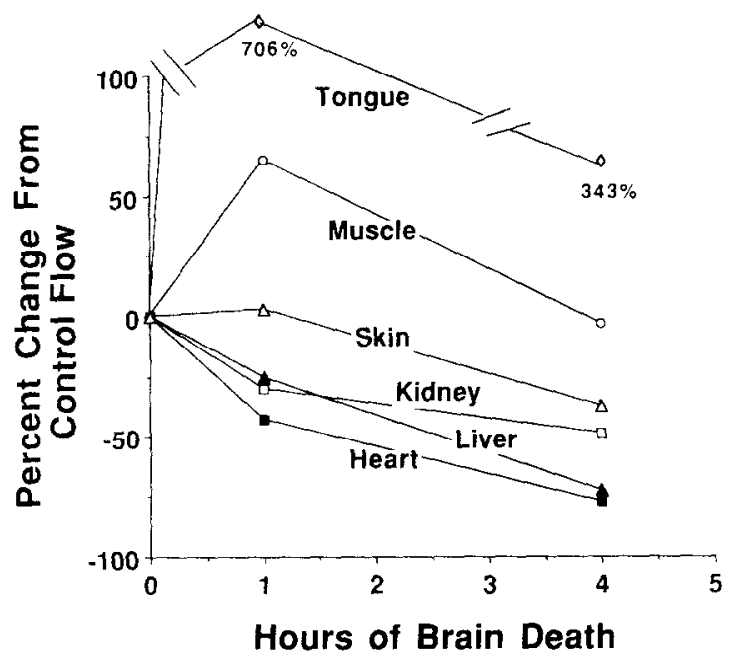

Fig 4. The percentage change of microsphere calculated control blood flow is graphed for the labeled tissues at the time points of the microsphere injections. The zero time point in this graph corresponds to the control microsphere injection prior to brain death. The reported organ flows correspond to specific tissues in Table 1: heart (left ventricular endocardial), liver (right lobe parenchymall, and kidney (right outer cortex).

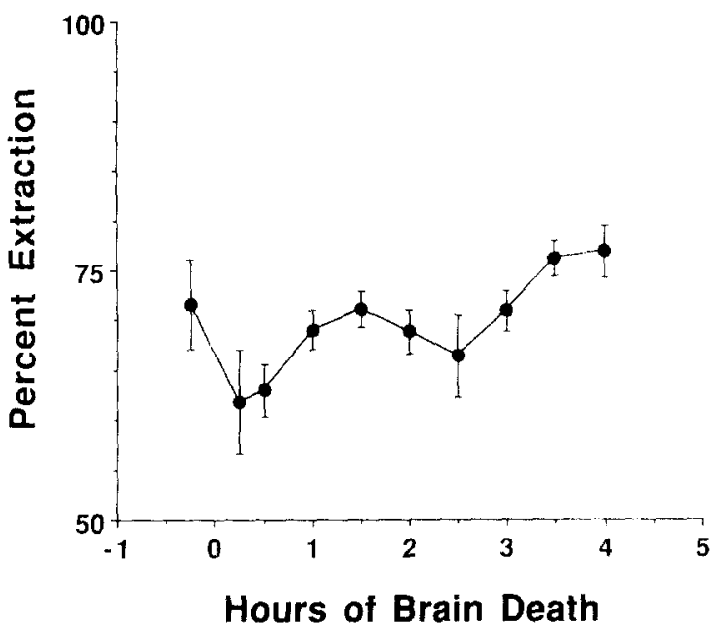

Fig 5. The percentage of coronary sinus oxygen extracted is graphed against the hours of brain death. The zero time point corresponds to the induction of brain death. The values were analyzed with an ANOVA test, and Scheffe's confidence intervals were used to determine the statistical significance of the differences. A level of $\boldsymbol{P} \leq .1$ was accepted as statistically significant. No statistical difference was found between the control and the other values.

sustained Cushing response. The evidence for this decrement was provided by a decrease of $\mathrm{CI}, \mathrm{SV}$, and $\mathrm{dP} / \mathrm{dt}$ after the induction of brain death. These changes were seen in the presence of a constant HR, constant PCWP or preload, and a decreasing MAP or afterload. This observed decrement of myocardial performance is consistent with other reports in the literature from Novitzky et al, ${ }^{1}$ Wicomb et al, ${ }^{4}$ and Sasaki et al, ${ }^{5}$ but leaves undefined the cause of this deterioration.

Myocardial ischemia/hypoxia or changes in contractility could be responsible for the decrement of performance. The latter hypothesis was suggested by a decrease of left ventricular $\mathrm{dP} / \mathrm{dt}$. While the $\mathrm{dP} / \mathrm{dt}$ is generally accepted to approximate the global contractile state, it is load dependent. Assessment of the contractile state requires a more rigorous methodology, such as the load-independent left ventricular pressure-volume loop analysis. ${ }^{10}$ Indeed, Sasaki et $\mathrm{al}^{5}$ reported a decrement of contractility using this technique in a different brain-dead dog model. However, the interpretation of Sasaki et al's study is limited on two accounts: a decrease of contractility was not seen in the majority of the animals in the study and the animals were maintained on inotropic therapy.

In the current study, the significant decrease 
of blood flow to the myocardial left ventricle and septum concomitant with the decrement of myocardial performance suggested that the myocardium might have been ischemic and that myocardial blood flow could have been limiting performance. Therefore, the coronary sinus oxygen extraction ratio was measured to test whether hypoxic ischemia was limiting performance. The myocardium normally extracts approximately $75 \%$ of the oxygen delivered; ischemic myocardium would be expected to extract a greater percentage of oxygen. Although the oxygen extraction tended to increase, it did not significantly increase or reach the predicted ischemic range values, failing to support the hypothesis and thus suggesting that in this brain-dead preparation the myocardium is not ischemic despite an MAP as low as $60 \mathrm{~mm} \mathrm{Hg}$. The myocardium may be deteriorating despite a normal coronary sinus oxygen extraction ratio if oxygen uptake or metabolism is reduced in parallel with blood flow. Coronary sinus or myocardial tissue $\mathrm{pH}$ measurements or lactate levels would be helpful in this setting. Indeed, Novitzky et al ${ }^{11}$ reported elevated tissue lactate after the induction of brain death; however, these findings were not correlated with oxygen delivery, extraction, or metabolism.

The microsphere studies were performed to provide a baseline of multiorgan blood flow and to investigate the potential of organ ischemia as a contributor to the deterioration of both the myocardium and the general preparation. It is notable that the microsphere-calculated flow values at 1 hour were statistically unchanged or decreased for all tissues with the exception of the skeletal muscle and tongue, while the calculated flow values to all organs decreased over the time interval from 1 to 4 hours. This initial increase in skeletal muscle and tongue flow suggested that the percentage decrease in resistance across these beds after brain death was greater than the percentage decrease in perfusion pressure. Although the actual magnitudes of these flow changes in $\mathrm{mL} / \mathrm{min} / 100 \mathrm{~g}$ of tissue were small, the total increase in flow to all the skeletal muscle was potentially a significant fraction of cardiac output in light of its large percentage of body mass. The increased distribution of cardiac output to these tissues after the initiation of brain death was likely inappropri- ate in light of their presumed decrement in metabolic needs in the absence of all neural inputs. Additionally, the tongue is the major thermoregulatory effector in the dog and thus could contribute significant thermal losses. The observed decrease in blood flow to both the kidney and the liver sections was notable for their relevance to transplantation, but the contribution to the decrement of the preparation was uncertain. The actual calculated flow values to the renal sections were not suggestive of ischemia; however, the effect on renal clearance was not determined. Similarly, the decrease in flow to the liver was relatively small, but it must be emphasized that the microsphere technique only measures hepatic arterial flow and not portal flow.

The maldistribution of cardiac output through systemic arteriovenous shunts was hypothesized as an additional potential mechanism for the deterioration of the preparation. This was suggested from pilot studies by a decrease in SVR and an increase of mixed venous oxygen saturation after the induction of brain death. Indeed, Cronenwett et $\mathrm{al}^{12}$ reported a $32 \%$ femoral artery regional shunt after hind limb sympathectomy. In our preparation, the percentage of cardiac output passing through systemic shunts was found to increase almost eightfold after the initiation of brain death. However, the absolute magnitude of the shunt remained less than $10 \%$ of cardiac output. Inherent in these calculations is error from the bronchial arterial flow; however, this contribution was presumed to be small ${ }^{9}$ and would overestimate the true shunt value. It is uncertain what contribution arteriovenous shunting of this small magnitude would make to the somatic deterioration of the preparation.

The explanation for the observed decrement of myocardial performance is unclear since it cannot be easily explained by either volume, myocardial hypoxic ischemia, or arteriovenous shunts. Novitzky et $a^{1}{ }^{1}$ reported a complex series of electrocardiographic changes after the induction of brain death. However, these changes were not seen in our study, thus making it difficult to invoke as a mechanism. Recently, acute hormonal insufficiency has been proposed as a potential mechanism for the deterioration. Novitzky et $a^{1}{ }^{1}$ reported decreases in the circu- 
lating stress hormones in a primate brain-dead model and emphasized the central role of thyroid hormone. They reported an improvement of performance after triiodothyronine supplementation in a working heart preparation using hearts excised from a brain-dead pig model. ${ }^{13}$ Furthermore, Novitzky et al ${ }^{11}$ reported the conversion from anaerobic to aerobic metabolism in the myocardium after triiodothyronine supplementation in the primate model. Despite the appeal, the potential role of an acute hypothyroid state has been controversial and it has not been documented in all clinical and experimental reports. ${ }^{14-20}$ Clearly, future studies are required to more fully document the changes in myocardial contractility and to clarify the role of thyroid hormone.

The preparation used in the present study afforded a severe, consistent, and reproducible model of the potential organ donor. The direct clinical applicability of the model, however, is limited on several accounts. The clinical declaration of brain death and the Cushing reflex can occur over minutes or take days to evolve. In this model, the induction of brain death took approximately 10 minutes, while the Cushing reflex was intentionally overridden and limited to 1 to 2 minutes. Clearly, a sustained Cushing reflex can play a significant role in exacerbating the deterioration of myocardial performance. ${ }^{2,3}$ The current study excludes this confounding influence. The use of anesthesia and the absence of associated trauma are further simplifications made to allow for a more controlled study. A final difference from the clinical setting is the extent of reduction in CNS blood flow. Our method for inducing brain death effectively stopped all CNS blood flow and, therefore, cortex, brain stem, and spinal cord function. ${ }^{6}$ However, the declaration of clinical brain death does not require the absence of spinal cord function, although it has been reported in $25 \%$ of cases by Ivan. ${ }^{2 !}$ Despite these recognized limitations, the model affords the ability to investigate the myocardial and hemodynamic changes that may contribute to the deterioration of the brain-dead dog in the absence of the Cushing reflex.

The present study illustrates several features intrinsic to the model and the potential organ donor. Brain death resulted in an essentially fixed HR presumably secondary to the loss of autonomic control. Previously published reports from our laboratory ${ }^{6}$ documented the absence of all baroreceptor response to pressor and depressor challenges in this model. This finding has been supported clinically by the inability to respond to vagolytic agents.22 This loss of chronotropic control limits the ability of the preparation to increase cardiac output. Additionally, the induction of brain death resulted in a marked decrease in SVR. Several explanations can be proposed to explain the decrease of SVR, including the redistribution of cardiac output through previously underperfused beds and potential circulating vasodilators; however, the most likely factor is a loss of neural control. The gradual increase of SVR in our study was surprising in the absence of CNS function. ${ }^{6}$ It suggests either a local control mechanism or circulating vasoconstrictors without CNS control. The absence of systemic acidosis supports the continued integrity of local control factors even at reduced MAP.

The induction of brain death leads to a decrement of myocardial performance. Indirect evidence suggests that this decrement is secondary to a decrease in myocardial contractility. The microsphere blood flow studies initially suggested that the decrement of performance may have been secondary to myocardial ischemia. However, this was not supported by a significant increase in the coronary sinus oxygen extraction ratio. Therefore, myocardial ischemic hypoxia cannot be the cause of the decrement in myocardial performance in this brain-dead dog preparation. Hormonal or metabolic factors other than blood flow or oxygen delivery must initiate the progressive deterioration of the heart in the brain-dead state.

\section{REFERENCES}

1. Novitzky D, Wicomb WN, Cooper DKC, et al: Electrocardiographic, hemodynamic, and endocrine changes occurring during experimental brain death in the chacma baboon. Heart Transplant Proc 4:63-69, 1984

2. Novitzky D, Cooper DKC, Rose AG, et al: Prevention of myocardial injury by pretreatment with verapamil hydrochloride prior to experimental brain death: Efficacy in a baboon model. Am J Emerg Med 5:11-18, 1987

3. Novitzky D, Wicomb WN, Cooper DKC, et al: Prevention of myocardial injury during brain death by total cardiac 
sympathectomy in the chacma baboon. Ann Thorac Surg 41:520-524, 1986

4. Wicomb WN, Cooper DKC, Lanza RP, et al: The effects of brain death and 24 hours' storage by hypothermic perfusion on donor heart function in the pig. $J$ Thorac Cardiovasc Surg 91:896-909, 1986

5. Sasaki H, Sada S, Beppu S, et al: Left ventricular pressure-volume relationships in brain-dead canine heartsPreoperative evaluation of donor hearts. Transplant Proc 21:2570-2572, 1989

6. Huber TS, D'Alecy LG: A simplified organ donor model produced by permanent complete CNS ischemia in dogs. J Crit Care 6:12-18, 1991

7. D'Alecy LG, Rose CJ, Sellers SA: Sympathetic modulation of hypercapnic cerebral vasodilation in dogs. Circ Res 45:771-785, 1979

8. Van Wylen DGL, D'Alecy LG: Regional blood flow distribution during the Cushing response: Alterations with adrenergic blockade. Am J Physiol 248:H98-H108, 1985

9. Heymann MA, Payne BD, Hoffman JE, et al: Blood flow measurements with radionuclide-labeled particles. Prog Cardiovasc Dis 20:55-79, 1977

10. Sagawa $\mathrm{K}$ : The ventricular pressure volume diagram revisited. Circ Res 43:677-687, 1978

11. Novitzky D, Cooper DKC, Morrell D, et al: Change from aerobic to anaerobic metabolism after brain death, and reversal following triiodothyronine therapy. Transplantation 45:32-36, 1986

12. Cronenwett JL, Zelenock GB, Whitehouse WM, et al: The effect of sympathetic innervation of canine muscle and skin blood flow. Arch Surg 118:420-424, 1983
13. Novitzky D, Wicomb WN, Cooper DKC, et al: Improved cardiac function following hormonal therapy in brain dead pigs: Relevance to organ donation. Cryobiology 24:1-10, 1987

14. Castellanos J, Tolcdo-Pereyra LH, Finkclstein I: Hormonal response to experimentally induced brain death. Transplant Proc 20:731-732, 1988

15. Finkelstein I, Toledo-Pereyra LH, Castellanos J: Physiologic and hormonal changes in experimentally induced brain dead dogs. Transplant Proc 9:4156-4158, 1987

16. Keogh AM, Howlett TA, Perry L, et al: Pituitary function in brain-stem dead organ donors: A prospective survey. Transplant Proc 20:729-730, 1988

17. Macoviack JA, McDougall IR, Bayer MF, et al: Significance of thyroid dysfunction in human cardiac allograft procurement. Transplantation 43:824-826, 1987

18. Montero JA, Mallol J, Alvarez F, et al: Biochemical hypothyroidism and myocardial damage in organ donors: Are they related? Transplant Proc 20:746-748, 1988

19. Rubertson KM, Hraniak IM, Gelb AW: Endocrine changes and hemodynamic stability after brain death. Transplant Proc 21:1197-1198, 1989

20. Wahlers T, Fieguth HG, Jurmann M, et al: Does hormone depletion of organ donors impair myocardial function after cardiac transplantation? Transplant Proc 20:792-794, 1988

21. Ivan LP: Spinal reflexes in cerebral death. Neurology 23:650-652, 1973

22. Vaghadia H: Atropine resistance in brain-dead organ donors. Anesthesiology 65:711-712, 1986 